Communications in Physics, Vol. 22, No. 3 (2012), pp. 213-221

\title{
NEUTRALITY EFFECTS ON THE PHASE STRUCTURE OF THE LINEAR SIGMA MODEL WITH THE NON-STANDARD SYMMETRY BREAKING TERM
}

\author{
TRAN HUU PHAT \\ Vietnam Atomic Energy Commission \\ NGUYEN VAN LONG \\ Gia Lai Junior College of Education \\ NGUYEN VAN THU \\ Department of Physics, Hanoi University of Education II \\ and \\ Institute for Nuclear Science and Technique
}

\begin{abstract}
The neutrality effects on the phase structure of the linear sigma model with the nonstandard symmetry breaking term is systematically studied by means of the Cornwall-JackiwTomboulis effective potential. The latter quantity is calculated in the improved Hartree-Fock approximation which preserves the Goldstone theorem and the thermodynamic consistency. Its results that in the region $\mu>m_{\pi}$, as a function of the temperature $T$, the pion condensate undergoes a second order phase transition, as a function of the isospin chemical $\mu$, it undergoes a first order phase transition. In the region $\mu<m_{\pi}$, the pion condensation is always a first order phase transition.
\end{abstract}

\section{INTRODUCTION}

At present it is well known that the phase structure of dense matter and compact stars is an interesting subject. Until now we are aware of only few points in the $T-\rho$ plane, namely, the vacuum $(T=0, \rho=0)$ and nuclear matter $\left(T=0, \rho=\rho_{0}=0.17 \mathrm{fm}^{-3}\right)$. Therefore, there are much experimental and theoretical efforts to get information about other regions. In recent years experimental studies are carried out through observing the matter produced in heavy-ion collisions at intermediate energies, in particular, the nuclear reactions induced by radioactive beams which offer a new opportunity to consider the isospin degree of freedom of asymmetric nuclear matter. As a consequence, the theoretical researches of Quantum Chromodynamics (QCD) at finite baryon density and isospin chemical potential are intensively implemented. It was shown that in the color-flavorlocked phase kaon condensation occurs at high baryon density and low temperature [1] and the lattice QCD simulation at finite isospin chemical potential [2-4] proved that there manifests the Bose-Einstein condensation of charged pions at high isospin density and low temperature. In [5] the phase diagram for pion condensation in QCD was suggested. In this connection, investigating simplified models of strongly interacting matter, such as the Nambu-Jona-Lasinio model [6-10], the linear sigma model [11-13], the chiral perturbation 
theory [14-16] etc., is very important for the understanding of matter states under different conditions. In our previous work [17] the kaon condensation was considered in the linear sigma model, where we developed a self-consistent approach involving the renormalization prescription, the thermodynamic consistency and the preservation of the Goldstone theorem. Besides, in [18], making use of this method we considered the phase structure of linear sigma model in the non-standard case of explicitly symmetry breaking basing on the Cornwall-Jackiw-Tomboulis (CJT) effective potential, but there was no electric neutrality constraint.

In this paper, we considered the neutrality effects on the phase structure of the linear sigma model with the non-standard symmetry breaking term by means of the CornwallJackiw-Tomboulis effective potential. The paper is structured as follows. In Section II, the calculation of renormalized CJT effective potential in [18] are resumed. In Section III the neutrality effects on the phase structure are numerically studied. The numerical computation is realized with the aid of Mathematica [20]. The conclusion and discussion are given in Section IV.

\section{RENORMALIZED CJT EFFECTIVE POTENTIAL}

The Lagrangian of the linear sigma model at finite isospin chemical potential reads

$$
\begin{aligned}
& L_{L S M}=\frac{1}{2}\left[\left(\partial_{\alpha} \sigma\right)^{2}+\left(\partial_{\alpha} \vec{\pi}\right)^{2}+2 i \mu\left(\pi_{1} \partial_{0} \pi_{2}-\pi_{2} \partial_{0} \pi_{1}\right)\right]-V, \\
& V=-\frac{m^{2}}{2}\left(\sigma^{2}+\vec{\pi}^{2}\right)-\frac{\mu^{2}}{2}\left(\pi_{1}^{2}+\pi_{2}^{2}\right)+\frac{\lambda^{2}}{4}\left(\sigma^{2}+\vec{\pi}^{2}\right)^{2} .
\end{aligned}
$$

According to [21] there are two types of explicit symmetry breaking

$$
L_{S B}^{(1)}=c \sigma,
$$

and

$$
L_{S B}^{(2)}=b \vec{\pi}^{2} .
$$

As we know, (2) is the standard case, which has been the research subject in most articles exploring the physical properties of the linear sigma model. To our understanding, the study of non-standard case justified by (3) is so far still absent and the goal of present paper is to remedy this gap. Therefore the Lagrangian we shall deal with is

$$
L=L_{L S M}+L_{S B}^{(2)},
$$

with

$$
m^{2}=\frac{m_{\sigma}^{2}}{2}>0, \lambda^{2}=\frac{m_{\sigma}^{2}}{2 f_{\pi}^{2}}>0, b=\frac{m_{\pi}^{2}}{2}
$$

in which $m_{\sigma}, m_{\pi}$ are respectively the masses of sigma and pion mesons in vacuum, and $f_{\pi}$ is the pion decay constant in vacuum.

As was shown in [21] the field operators $\sigma$ and $\pi_{i},(i=1,2,3)$ develop the following expectation values in the ground state

$$
\langle\sigma\rangle=u=f_{\pi},\left\langle\pi_{i}\right\rangle=v=0,
$$


for $\mu<m_{\pi}$ and

$$
\langle\sigma\rangle=u=0,\left\langle\pi_{1}\right\rangle=v \neq 0,\left\langle\pi_{2,3}\right\rangle=0
$$

for $\mu>m_{\pi}$.

In the tree approximation $u$ and $v$ are determined from the minimization of the potential energy of (4), namely, they fulfill the equations

$$
\begin{aligned}
& {\left[-m^{2}+\lambda^{2}\left(u^{2}+v^{2}\right)\right] u=0,} \\
& {\left[-\mu^{2}-m^{2}+\lambda^{2}\left(u^{2}+v^{2}\right)+m_{\pi}^{2}\right] v=0,}
\end{aligned}
$$

which yield

$$
u=f_{\pi}, v=0,
$$

for $\mu<m_{\pi}$ and

$$
u=0, v^{2}=\frac{m^{2}+\mu^{2}-m_{\pi}^{2}}{\lambda^{2}}
$$

for $\mu>m_{\pi}$.

Realizing the shifts on field operators

$$
\sigma \rightarrow u+\sigma, \pi_{1} \rightarrow v+\pi_{1}, \pi_{2,3} \rightarrow \pi_{2,3},
$$

and then inserting (8) into (4) it is derived the interaction Lagrangian

$$
\begin{aligned}
L_{I}= & {\left[m^{2} u-\lambda^{2} u\left(u^{2}+v^{2}\right)\right] \sigma+\left[m^{2} v+\mu^{2} v-\lambda^{2} v\left(u^{2}+v^{2}\right)-2 b v\right] \pi_{1} } \\
& -\lambda^{2}\left(\sigma^{2}+\vec{\pi}^{2}\right)\left(u \sigma+v \pi_{1}\right)-\frac{\lambda^{2}}{4}\left(\sigma^{2}+\vec{\pi}^{2}\right)^{2},
\end{aligned}
$$

and the inverse propagators

$$
\begin{aligned}
& i D_{0}^{-1}(k ; v, u)=\left(\begin{array}{cc}
\omega_{n}^{2}-k^{2}+m_{1}^{2} & 4 i \mu \omega_{n} \\
-4 i \mu \omega_{n} & \omega_{n}^{2}-k^{2}+m_{2}^{2}
\end{array}\right), \\
& i G_{0}^{-1}(k ; v, u)=\left(\begin{array}{cc}
\omega_{n}^{2}-k^{2}+m_{3}^{2} & 0 \\
0 & \omega_{n}^{2}-k^{2}+m_{4}^{2}
\end{array}\right),
\end{aligned}
$$

with

$$
\begin{aligned}
& m_{1}^{2}=\mu^{2}+m^{2}-\lambda^{2} u^{2}-3 \lambda^{2} v^{2}-m_{\pi}^{2}, m_{3}^{2}=m^{2}-3 \lambda^{2} u^{2}-\lambda^{2} v^{2}, \\
& m_{2}^{2}=\mu^{2}+m^{2}-\lambda^{2} u^{2}-\lambda^{2} v^{2}-m_{\pi}^{2}, m_{4}^{2}=m^{2}-\lambda^{2} u^{2}-\lambda^{2} v^{2}-m_{\pi}^{2} .
\end{aligned}
$$

Combining (6), (7) and (10) indicates that there appears a gapless mode corresponding to $\pi_{2}$.

Basing on (9), (10) and (11) we dealt with the CJT effective potential in the doublebubble approximation, which also is called the Hartree-Fock approximation [19]. Hence, there are two distinct Hartree-Fock approximations: the one that respects the Goldstone theorem is called the improved Hartree-Fock (IHF) approximation and the other related to the absence of the Goldstone theorem is called briefly the usual HF approximation. In [18] we have established the renormalized CJT effective potential in IHF approximation, 


$$
\begin{aligned}
\widetilde{V}_{\beta}^{C J T}(u, v ; D, G)= & -\frac{m^{2}}{2}\left(u^{2}+v^{2}\right)-\frac{\mu^{2}}{2} v^{2}+\frac{\lambda^{2}}{4}\left(u^{2}+v^{2}\right)^{2}+b v^{2} \\
& +\frac{1}{2} \int_{\beta} \operatorname{tr}\left\{\ln D^{-1}(k)+\ln G^{-1}(k)+D_{0}^{-1}(k, v) D+G_{0}^{-1}(k, v) G-2.11\right\} \\
& +\frac{\lambda^{2}}{4} P_{11}^{2}+\frac{\lambda^{2}}{4} P_{22}^{2}+\frac{3 \lambda^{2}}{2} P_{11} P_{22}+\frac{3 \lambda^{2}}{4} Q_{11}^{2}+\frac{3 \lambda^{2}}{4} Q_{22}^{2}+\frac{\lambda^{2}}{2} Q_{11} Q_{22} \\
& +\frac{\lambda^{2}}{2}\left(P_{11} Q_{11}+P_{11} Q_{22}+P_{22} Q_{11}+P_{22} Q_{22}\right),
\end{aligned}
$$

which generates the gap equations and the inverse propagators as follows.

- The gap equations

$$
\begin{aligned}
& -m^{2}+\lambda^{2}\left(u^{2}+v^{2}\right)+\widetilde{\Sigma}_{\sigma}=0, \\
& -\mu^{2}-m^{2}+m_{\pi}^{2}+\lambda^{2}\left(u^{2}+v^{2}\right)+\widetilde{\Sigma}_{\pi_{2}}=0 .
\end{aligned}
$$

-The inverse propagators

$$
\begin{gathered}
D^{-1}(k)=\left(\begin{array}{cc}
\omega_{n}^{2}+k^{2}+M_{1}^{2} & -4 \mu \omega_{n} \\
4 \mu \omega_{n} & \omega_{n}^{2}+k^{2}
\end{array}\right), \\
G^{-1}(k)=\left(\begin{array}{cc}
\omega_{n}^{2}+k^{2}+M_{3}^{2} & 0 \\
0 & \omega_{n}^{2}+k^{2}+M_{4}^{2}
\end{array}\right),
\end{gathered}
$$

with

$$
\begin{gathered}
\int_{\beta} f(k)=\frac{1}{\beta} \sum_{n=-\infty}^{+\infty} \int \frac{d^{3} k}{(2 \pi)^{3}} f\left(\omega_{n}, k\right), \omega_{n}=\frac{2 \pi n}{\beta}, \beta=\frac{1}{T} . \\
P_{a a}=\int_{\beta} D_{a a}, Q_{b b}=\int_{\beta} G_{b b} . \\
M_{1}^{2}=-\mu^{2}-m^{2}+3 \lambda^{2} v^{2}+\lambda^{2} u^{2}+m_{\pi}^{2}+\widetilde{\Sigma}_{\pi_{1}} \\
M_{3}^{2}=-m^{2}+\lambda^{2} v^{2}+3 \lambda^{2} u^{2}+\widetilde{\Sigma}_{\sigma} \\
M_{4}^{2}=-m^{2}+\lambda^{2} v^{2}+\lambda^{2} u^{2}+m_{\pi}^{2}+\widetilde{\Sigma}_{\pi_{3}}
\end{gathered}
$$

and

$$
\begin{aligned}
& \widetilde{\Sigma}_{\pi_{1}}=\lambda^{2}\left(P_{11}+3 P_{22}+Q_{11}+Q_{22}\right), \\
& \widetilde{\Sigma}_{\pi_{2}}=\lambda^{2}\left(3 P_{11}+P_{22}+Q_{11}+Q_{22}\right), \\
& \widetilde{\Sigma}_{\sigma}=\lambda^{2}\left(3 Q_{11}+Q_{22}+P_{11}+P_{22}\right), \\
& \widetilde{\Sigma}_{\pi_{3}}=\lambda^{2}\left(Q_{11}+3 Q_{22}+P_{11}+P_{22}\right) .
\end{aligned}
$$

The Goldstone theorem is immediately deduced from Eq. (14a),

$$
\operatorname{det} D^{-1}(k)=0
$$


yielding

$$
\omega_{\pi_{2}} \approx \sqrt{-1+\frac{8 \mu^{2}}{M_{1}^{2} / 2+8 \mu^{2}}} k \text { as } k \rightarrow 0 .
$$

The conventional (one-particle-irreducible) effective potential is straightforwardly derived by inserting the SD equations (20) into (12),

$$
\begin{aligned}
V_{\beta}(u, v)= & -\frac{m^{2}}{2}\left(u^{2}+v^{2}\right)-\frac{\mu^{2}}{2} v^{2}+\frac{\lambda^{2}}{4}\left(u^{2}+v^{2}\right)^{2}+b v^{2} \\
& +\frac{1}{2} \int_{\beta} \operatorname{tr}\left\{\ln D^{-1}(k)+\ln G^{-1}(k)\right\}-\frac{\lambda^{2}}{4}\left(P_{11}^{2}+P_{22}^{2}+6 P_{11} P_{22}\right) \\
& -\frac{\lambda^{2}}{4}\left(3 Q_{11}^{2}+3 Q_{22}^{2}+2 Q_{11} Q_{22}\right) \\
& -\frac{\lambda^{2}}{2}\left(P_{11} Q_{11}+P_{11} Q_{22}+P_{22} Q_{11}+P_{22} Q_{22}\right) .
\end{aligned}
$$

Next let us prove the thermodynamic consistency. To this end, we note that the pressure $P$ is determined by

$$
P=\left.\left(-\widetilde{V}_{\beta}^{C J T}\right)\right|_{\text {at minimum }} .
$$

Then the isospin density is found

$$
\rho=\frac{\partial P}{\partial \mu}=\mu\left(v^{2}+P_{11}+P_{22}\right)
$$

Eq. (17) expresses exactly the thermodynamic consistency

$$
\rho=\mu \operatorname{Tr} D(x, y) .
$$

\section{ELECTRIC NEUTRALITY AND PHASE STRUCTURE}

We know that the pion density $\rho$ consists of the contributions from both charged pions,$\pi^{+}$and $\pi^{-}$. Therefore, in order to neutralize the whole system in broken phase we must invoke the participation of leptons. For simplicity we consider only the case when electrons and positrons are present. Then the electric charge density reads

$$
n_{Q}=\alpha \rho-(1-\alpha) \rho+2 \sum_{e=+1,-1} e \int \frac{d^{3} \vec{k}}{(2 \pi)^{3}} f_{F}\left(\varepsilon^{e}\right),
$$

where $\alpha \rho$ and $(\alpha-1) \rho$ denote respectively the contributions of $\pi^{+}$and $\pi^{-}$to the condensate; $0 \leq \alpha \leq 1 ; f_{F}$ is the Fermi distribution

$$
f_{F}(x)=\frac{1}{e^{x / T}+1}
$$

the excitation energies are

$$
\varepsilon^{e}=\sqrt{\vec{k}^{2}+m_{e}^{2}}-e \mu_{Q},
$$

with $\mu_{Q}$ and $m_{e}$ being electric chemical potential and electron mass, respectively. 
The electric neutrality requires

$$
n_{Q}=0
$$

or

$$
\alpha \rho-(1-\alpha) \rho+\left.2 \sum_{e=+1,-1} e \int \frac{d^{3} \vec{k}}{(2 \pi)^{3}} f_{F}\left(\varepsilon^{e}\right)\right|_{\mu_{Q}=\mu}=0 .
$$

Eq. (18) is a constraint imposed on $T$ and $\mu$.

We assume that there is no lepton chemical potential and we also assume that electron mass is negligibly small in what follows.

Before proceeding to the numerical study let us remark that the divergent integrals are regularized, therefore, in addition to the model parameters, they introduced to the theory different scale parameter $\gamma_{0}$ (see [18]). In order to determine the values of this scale parameter it is required that

$$
u(T=0, \mu=0)=f_{\pi} .
$$

Taking the pion and sigma masses $m_{\pi}=138 \mathrm{MeV}, m_{\sigma}=500 \mathrm{MeV}$ and the pion decay constant $f_{\pi}=93 \mathrm{MeV}$ then (19) gives $\gamma_{0}=487.4 \mathrm{MeV}$.

At present we are ready to carry out the numerical computation for two different regions of $\mu$.

\section{III.1. Region $\mu>m_{\pi}$}

In this region $u=0$ and $v$ is given by the gap equation

$$
-\mu^{2}-m^{2}+m_{\pi}^{2}+\lambda^{2} v^{2}+\widetilde{\Sigma}_{\pi_{2}}=0,
$$

or

$$
v^{2}=\frac{m^{2}+\bar{\mu}^{2}-m_{\pi}^{2}}{\lambda^{2}}
$$

where we define the effective isospin chemical potential $\bar{\mu}^{2}=\mu^{2}-\widetilde{\Sigma}_{\pi_{2}}$.

In Fig. 1 is shown the evolution of pion condensate as function of $T$. For comparison, we also present the graph of $v(T, \mu)$ at $\mu=200 \mathrm{MeV}$ for the case when the system is not constrained by (18). It is easily seen that the pion condensation is second order phase transition and the critical temperature is influenced by the electric neutrality constraint. The evolution of pion condensate against $\mu$ is plotted in Fig. 2 for several values of $\alpha$. The phase transition is first order everywhere corresponding to $\mu \geq m_{\pi}$.

III.2. Region $\mu<m_{\pi}$

In this case $v=0$ and the gap equation (13b) turns out to be

$$
-m^{2}+\lambda^{2} u^{2}+\widetilde{\Sigma}_{\sigma}=0,
$$

or

$$
u^{2}=\frac{m^{2}-\widetilde{\Sigma}_{\sigma}}{\lambda^{2}} .
$$

Solving self-consistently Eq. (21) together with the neutrality condition (18) we obtain the evolution of pion condensate as a function of the temperature in Fig. 3 and the 


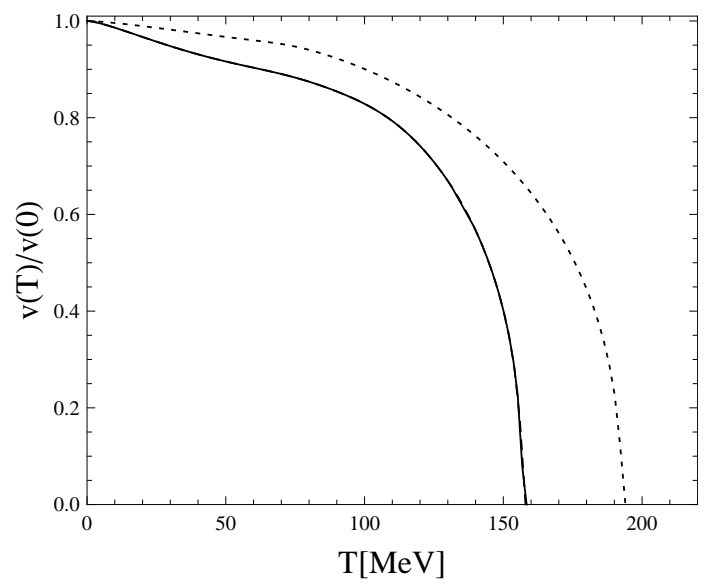

Fig. 1. The evolution of pion condensate as a function of the temperature normalized to the condensate at zero temperature. The solid line (dashed line) corresponds to the case when (18) is included (not included and $\mu=200 \mathrm{MeV}$ ).

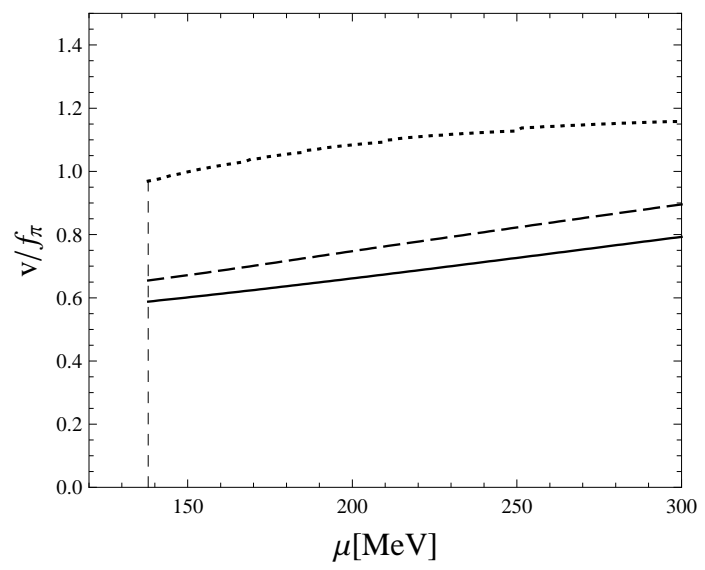

Fig. 2. The evolution of pion condensate as a function of the isospin chemical potential. The solid, dashed and dotted lines correspond to $\alpha=0,1 / 4,1 / 2$, respectively.

$\mu$ dependence of pion condensate for several of $\alpha$ in Fig. 4. From the figures we recognize that the phase transition is first order everywhere and moreover, the pion condense at $\mu=0$. For this case, the critical temperature is not almost influenced by the electric neutrality constraint.

\section{CONCLUSION}

In this paper the phase structure of the linear sigma model with the non-standard symmetry breaking term was studied by means of the Cornwall-Jackiw-Tomboulis effective 


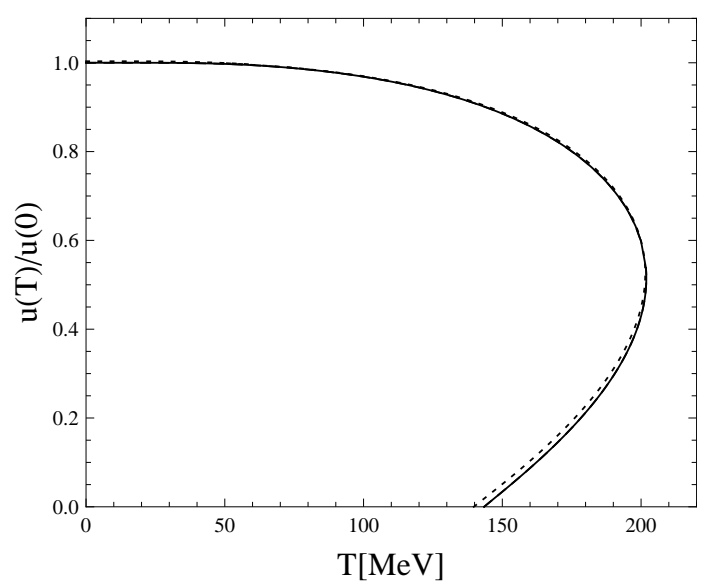

Fig. 3. The evolution of chiral condensate as a function of the temperature normalized to the condensate at zero temperature. The solid line (dashed line) corresponds to the case when (18) is included (not included with $\mu=100 \mathrm{MeV}$ ).

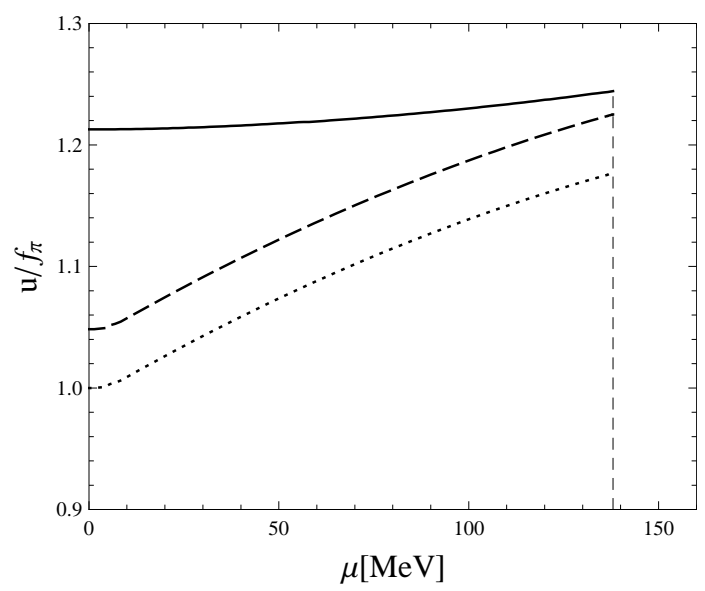

Fig. 4. The evolution of chiral condensate as a function of the isospin chemical potential. The solid, dashed and dotted lines correspond to $\alpha=0,1 / 4,1 / 2$, respectively.

potential. We investigated in detail the neutrality effects on the phase structure and found the main results as follows.

1- The electric neutrality constraint was established.

2- It was proved that in the region $\mu>m_{\pi}$, as a function of $T$, the pion condensation is second order phase transition, whereas, as a function of $\mu$, it become first order.

3- In the region $\mu<m_{\pi}$, the pion condensation is always a first order phase transition. 
In comparison with the case when the electric neutrality constraint is not included [18], it is clear that the neutrality condition evidently affects the phase transition in the region $\mu>m_{\pi}$.

\section{ACKNOWLEDGMENT}

N.V.Thu would like to express his sincere thanks to the Vietnam Atomic Energy Commission and the Institute of Nuclear Science and Technique for the hospitality during his PhD study.

This paper is financially supported by the Vietnam National Foundation for Science and Technology Development (NAFOSTED).

\section{REFERENCES}

[1] M. G. Alford, K. Rajagopal, F. Wiczek, Nucl. Phys. B 537 (1999) 443.

[2] J. B. Kogut, D. K. Sinclair, Phys. Rev. D 64 (2001) 034508; 70 (2004) 094501.

[3] P. de Forcrand, M. A. Stephanov, U. Wenger, PoS (Lattice 2007) 237.

[4] S. Gupta, "Critical Behavior in QCD at Finite Isovector Chemical Potential", arXiv:hep-lat/0202005.

[5] D. T. Son, M. A. Stephanov, Phys. Rev. Lett. 86 (2001) 592.

[6] L. He, Phys. Rev. D 82 (2010) 096003.

[7] A. Barducci, R. Casalbuoni, G. Pettini, L. Ravagli, Phys. Rev. D 71 (2005) 016011.

[8] L. He, M. Jin, P. Zhuang, Phys. Rev. D 71 (2005) 116001.

[9] L. He, P. Zhuang, Phys. Lett. B 615 (2005) 93.

[10] M. Frank, M. Buballa, M. Oertel, Phys. Lett. B 562 (2003) 221.

[11] J. O. Andersen, T. Brauner, Phys. Rev. D 78 (2008) 014030.

[12] J. O. Andersen, Phys. Rev. D 75 (2007) 065011.

[13] O. Scavenius, A. Mocsy, I. N. Mishustin, D. H. Rischke, Phys. Rev. C 64 (2001) 045202.

[14] M. C. Birse, T. D. Cohen, J. A. McGovern, Phys. Lett. B 516 (2001) 27.

[15] J. B. Kogut, D. Toublan, Phys. Rev. D 64 (2001) 034007.

[16] M. Loewe, Villavicencio, Phys. Rev. D 70 (2004) 074005.

[17] T. H. Phat, N. V. Long, N. T. Anh, L. V. Hoa, Phys. Rev. D 78 (2008) 105016.

[18] T. H. Phat, N. V. Thu, J. Phys. G: Nucl. Part. Phys. 38 (2011) 045002.

[19] G.Amelia-Camelia, S. Y. Pi, Phys. Rev. D 47 (1993) 2356.

[20] S. Wolfram, The Mathematica Book, 5th edition, 2003 Wolfram Media/Cambridge University Press.

[21] D. K. Campell, R. F. Dashen, J. T. Manassah, Phys. Rev. D 12 (1975) 979.

Received 30 September 2011. 